Subscriber access provided by Caltech Library

\title{
Article
}

\section{Mechanisms of diffusion in associative polymer networks: evidence for chain hopping}

Peter B Rapp, Ahmad K. Omar, Bradley R. Silverman, Zhen-Gang Wang, and David A. Tirrell

J. Am. Chem. Soc., Just Accepted Manuscript • DOI: 10.1021/jacs.8b07908 • Publication Date (Web): 01 Oct 2018

Downloaded from http://pubs.acs.org on October 1, 2018

\section{Just Accepted}

"Just Accepted" manuscripts have been peer-reviewed and accepted for publication. They are posted online prior to technical editing, formatting for publication and author proofing. The American Chemical Society provides "Just Accepted" as a service to the research community to expedite the dissemination of scientific material as soon as possible after acceptance. "Just Accepted" manuscripts appear in full in PDF format accompanied by an HTML abstract. "Just Accepted" manuscripts have been fully peer reviewed, but should not be considered the official version of record. They are citable by the Digital Object Identifier (DOI®). "Just Accepted" is an optional service offered to authors. Therefore, the "Just Accepted" Web site may not include all articles that will be published in the journal. After a manuscript is technically edited and formatted, it will be removed from the "Just Accepted" Web site and published as an ASAP article. Note that technical editing may introduce minor changes to the manuscript text and/or graphics which could affect content, and all legal disclaimers and ethical guidelines that apply to the journal pertain. ACS cannot be held responsible for errors or consequences arising from the use of information contained in these "Just Accepted" manuscripts. 


\section{INTRODUCTION}

Polymer chains can associate through non-covalent interactions (e.g., by hydrogen bonding, ${ }^{1-4}$ metal-ligand coordination ${ }^{3,}$ ${ }^{5-10}$ or hydrophobic forces ${ }^{1,11-14}$ ) to form reversible networks. Within such networks, clusters of associative domains serve as transient junctions. ${ }^{15}$ The dynamic nature of the junctions permits diffusive transport of chains throughout the network and facilitates spontaneous reorganization or "healing" of the network in response to mechanical damage. Reversible polymer networks have been proposed for application in tissue engineering, ${ }^{16-17}$ controlled drug delivery, ${ }^{18}$ and organic electronics and battery technology. ${ }^{19-20}$ Understanding the factors that control polymer diffusion in reversible networks is important for optimizing material performance in these settings, and for elucidating the principles that govern macromolecular transport in biological systems. For example, eukaryotic cells utilize networks of non-covalent interactions to regulate protein transport into the nucleus, ${ }^{21-22}$ and to control the localization of growth factors and cytokines in the extracellular matrix. ${ }^{23}$

Previous experimental ${ }^{5-7,11,13,24-26}$ and theoretical ${ }^{11,15,27-30}$ investigations of associative polymer networks have sought to relate their bulk physical properties (viscosity, elastic modulus, relaxation rate) to the underlying structural and dynamical configurations of the constituent chains. When the chains are not significantly interpenetrated (i.e., in the unentangled regime), network stress relaxation is typically attributed to chain disengagement from the junctions. Although this simple single-chain picture serves as the foundation for several successful rheological models, ${ }^{11,30-31}$ the physical basis for self-diffusion in associative networks is decidedly less clear. Despite nearly 30 years of conceptual development, ${ }^{27,32}$ no single mechanistic picture of how the constituent chains move has found quantitative experimental validation. This stands in contrast to our more complete understanding of self-diffusion in unassociative polymer solutions, for which mechanisms such as reptation find strong experimental support. ${ }^{33-36}$

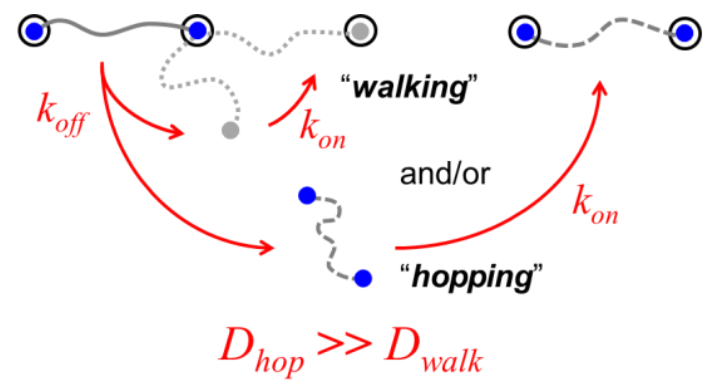

Figure 1. Diffusive migration of associative polymer chains through reversible networks can occur via partial or complete disengagement of the associative segments, i.e. by "walking" or "hopping". Here we provide evidence that $D_{\text {hop }} \gg D_{\text {walk }}$ in model networks.

For diffusion in the unentangled regime, two complementary mechanistic schemes have been predominantly invoked. If a chain disengages from the network completely such that it temporarily has no bound segments, it may diffuse freely over relatively large distances before rebinding (we refer to this process as "hopping", Figure 1). Alternatively, center-of-mass translation of the chain may be achieved by stepwise dissociation and reassociation of individual chain segments (in a process akin to "walking"), without the chain ever becoming completely untethered from the network. Baxandall considered the diffusion 
of single multisticker chains reversibly interacting with a meanfield (structureless) network, predicting an ensemble of walking modes. ${ }^{27}$ The walking diffusivity was proportional to the sticker dissociation rate, and inversely proportional to the number of stickers (effectively Rouse-like). Rubinstein and Semenov subsequently proposed a "sticky Rouse" picture which postulated that the bound chains are carried along in large multichain clusters. ${ }^{15}$ Recent simulations ${ }^{37}$ indicate that such cluster diffusion may become particularly important when the network is under steady shear, but its role in quiescent-state relaxation remains unclear. Both theoretical treatments disregard hopping, i.e., the fraction of free chains is assumed to be negligible. Olsen and co-workers invoked a form of bound diffusivity along with chain hopping to rationalize anomalous "super-diffusive" behavior within associative networks, wherein the effective diffusion coefficient appears to increase with time over a finite length-scale. ${ }^{10,13}$ Recently they proposed transient escape from network junctions, corresponding to a transition between walking and hopping, as the molecular origin of this intriguing phenomenon. ${ }^{32}$

The present study is motivated by our earlier observation that the sequential binding of the two ends of a telechelic polymer to a reversible network appears "asymmetric", i.e. the first association is stronger than the second, despite identical molecular properties of the terminal associative domains. ${ }^{25}$ We now demonstrate that marked differences between the intrinsic and effective binding strengths of associative domains on multisticker chains are a general feature of unentangled reversible networks. Whereas the intrinsic binding strength of a sticker is set by its molecular properties, the effective binding strength of the sticker is sensitive to the local structure of the network and is attenuated by the presence of other concurrently bound stickers on the same chain. The origin of this effect is a strong entropic penalty imposed on chain entry into conformationally restricted states during sequential binding events. A given chain must sacrifice rich sets of conformations in order to bind multiple junctions simultaneously. This constraint significantly amplifies the fraction of free (hopping) chains and diminishes as the ratio of the chain size to the network size increases.

We first incorporate these ideas into a simple thermodynamic model that predicts the fraction of chains that are free to hop as a function of tunable network and molecular properties. We then test our model predictions with a large set of self-diffusivity measurements obtained in telechelic associative protein hydrogels and find consistent agreement with the model. Surprisingly, hopping is the dominant mode of diffusion despite the large enthalpic penalty for dissociation. Furthermore, hopping remains a major diffusion mechanism for multisticker chains with as many as five stickers. These results provide new insight into the chain transport dynamics of an important class of polymeric materials.

\section{THEORY}

Network model. We consider associative networks in the dynamic regime where chain dissociation is much slower than the characteristic Rouse relaxation of the constituent chains (typical for most experimental associative networks). We model the network as an ensemble of multisticker chains having $S$ total associative domains ("stickers") that are equally-spaced along the chain, with stickers capping the ends of the chain. A chain can partition into $i \leq S$ bound states, where $i=0,1,2 \ldots S$ represents the total number of stickers bound to the network. One can define corresponding equilibrium constants $K_{i}$ (where $K_{i}$ is the binding constant characterizing the transition from $i-1$ to $i$ bound stickers) that describe how the chain distributes among these various states. For example, telechelic chains have two terminal stickers $(S=2)$ and can be partitioned into three sequential states (Figure 2A). In the free state $f$, neither chain end is bound to the network $(i=0)$. By reversible association with the network, the chain may transition into either the dangle state $d$ (one end bound, $i=1$ ) or the bound state $b$ (both ends bound, $i=2$ ). Two species compose the bound state of telechelic chains: bridges $(B)$, where the chain ends are bound to two different junctions, and loops $(L)$, where both ends converge on the same junction. Conversion among these three states is controlled by two equilibrium constants $K_{1}=[d] /[f]$ and $K_{2}=[B] /[d]+[L] /[d]=K_{B}+K_{L}$.

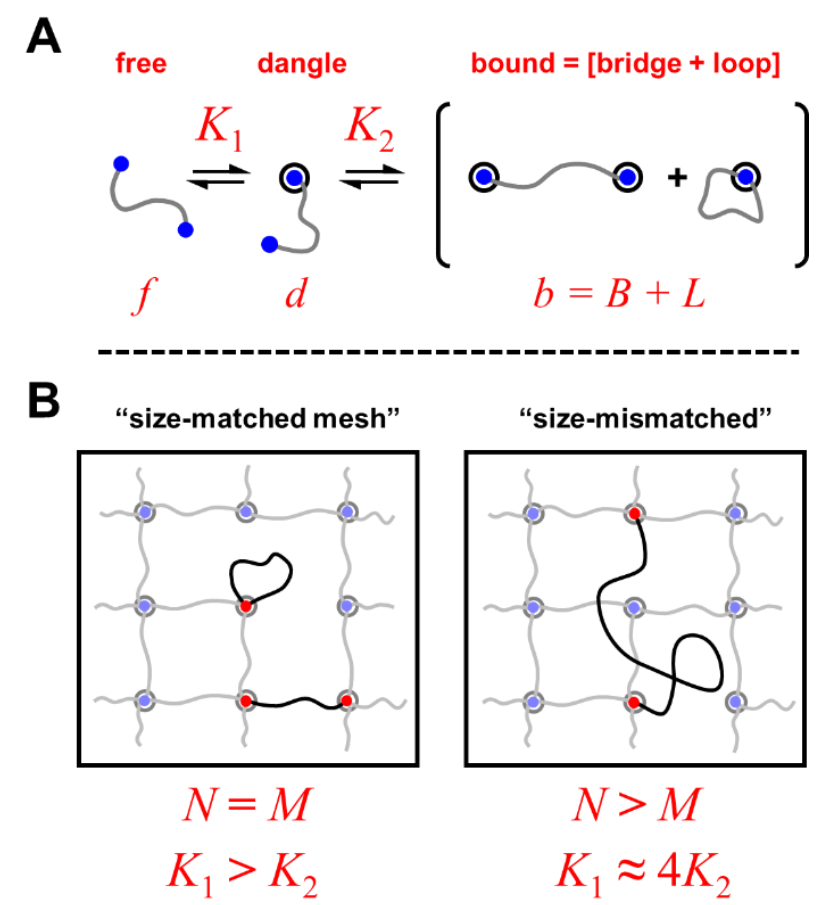

Figure 2. Schematic representation of telechelic chain partitioning in reversible networks. (A) Two equilibrium constants $K_{1}$ and $K_{2}$ control chain partitioning among three sequential states: free $(f)$, dangle $(d)$ and bound $(b)$. The bound state consists of bridges $(B)$ and loops $(L)$. (B) Local network structure affects the binding equilibrium of telechelic chains. When the chain size $(N)$ and mesh size $(M)$ are the same, an entropic penalty for bridge formation manifests as binding asymmetry $\left(K_{1}>K_{2}\right)$. As the chains become larger than the mesh, the difference in binding strengths decreases to the mean-field limit $\left(K_{1} \approx 4 K_{2}\right)$.

Previous approaches to modeling reversible networks consider chains interacting with a mean-field background and neglect spatial correlations between junctions (i.e., they envision a structureless network). ${ }^{15,} 27,32$ Overlooking local network structure in this way fails to capture an essential loss of entropy upon binding. The following theoretical considerations suggest that this entropic penalty attenuates each $K_{i}$ of a multisticker chain for all but the first association, leading to an effective network binding strength that is much smaller than would be expected from the mean-field approach. For telechelic chains, this manifests as binding asymmetry, with $K_{1}>K_{2}$ arising from conformational restrictions imposed on the bridge state. Simple thermodynamic considerations predict that this difference in 
binding strengths decreases as the chain size $(N)$ becomes large relative to the characteristic mesh size $(M)$ of the network (Figure 2B). In the limit of $N \gg M$, the chains begin to perceive the network as "mean-field-like" such that $K_{1} \approx 4 K_{2}$.

Statistical mechanics of chain binding. Each equilibrium constant $K_{i}$ described in the preceding section may be formally defined as the ratio of the number of chain configurations with $i$ bound stickers to the number with $i-1$ bound stickers, weighted by the binding energy. The equilibrium constants are obtained by equating the chemical potentials of the chains in each state (i.e., $\mu_{i}=\mu_{i-1}$ ). Neglecting interchain interactions apart from junction binding, one readily finds:

$$
K_{i}=\frac{Z_{i}}{Z_{i-1}} \exp (-\beta \Delta E)
$$

where $Z_{i}$ represents all possible configurations of a chain with $i$ bound stickers, $\beta=1 / k_{B} T$, and $\Delta E$ is the energy released upon binding of one of the stickers to a network junction. We model individual chains as consisting of $N$ statistical segments each of Kuhn length $b_{k}$. We consider probe chains with end-toend distance $R_{\text {probe }}=b_{k} N^{1 / 2}$ within a network of characteristic mesh size (inter-junction spacing) $R_{\text {mesh }} \sim M^{1 / 3}$, where $M$ is the number of statistical segments in the chains that compose the network. The latter scaling arises under the condition that as the network chains increase in size, the total concentration of polymer remains fixed such that the junction density linearly decreases with $M$. For telechelic polymers, the integral $Z_{0} \sim \Omega_{f}\left(N, b_{k}\right)=\int P\left(\mathbf{R} ; N, b_{k}\right) d \mathbf{R}$ counts the number of accessible configurations in the free (unbound, $i=0$ ) state to within a constant prefactor. This prefactor is inconsequential, as it may be absorbed into a reference chemical potential. ${ }^{38} P(\mathbf{R})$ represents the normalized end-to-end vector probability density function of the free probe chains, and as such, $Z_{0}$ is taken to be unity without loss of generality.

When one end of a telechelic chain attaches to a network junction (in a transition from free to dangle, $i=1$ ), the chain energy changes by an amount $\Delta E$. This transition entails a loss of translational entropy, as a dangling chain is restricted to a small fraction $\phi_{j}$ (proportional to the junction density) of the total system volume. However, the untethered chain end may still explore the local space around the junction. Additional losses in conformational entropy may therefore be neglected, provided the junction itself is small and the dangling chain does not "feel" the presence of other bound chains $\left(1-\phi_{j} \approx 1\right)$. These considerations suggest $Z_{1} \approx 2 \phi_{j} \Omega_{f}$, where the factor of two arises because a chain can occupy the dangle state by engaging either one of its ends (i.e., there are twice as many configurations of a telechelic chain with one sticker bound as for a "monochelic" chain with its only sticker bound ${ }^{39}$ ), and these two configurations are degenerate. From eq. 1, we obtain $K_{1}=2 \phi_{j} \exp (-\beta \Delta E)$.

The chain faces a new challenge during the second binding event (transition from dangle to bridge, $i=2$ ). In order to form a bridge, the untethered chain end must bind to a new junction some (vector) distance $\mathbf{R}$ away from the chain end that is already bound. The probability of finding this second junction $d e$ pends on the local structure of the network. Whereas a dangling chain has no external constraint imposed on its end-to-end distance, bridged chain conformations in which the two chain ends do not overlap with two different junctions cannot exist. Significant conformational entropy may therefore be lost during bridge formation, provided the network junctions tend to be separated by some characteristic distance $R_{m e s h}$. Although gels are isotropic and generally lack long-range order, local order (i.e., spatial correlation between junctions) is expected as a natural consequence of gelation: the network junctions cannot get too close to each other without placing elastic stress on neighboring chains. ${ }^{25}$

We formalize this network-imposed entropic constraint by restricting the second end of a bridged chain to a significantly reduced volume fraction $\phi_{j} g\left(\mathbf{R} ; R_{\text {mesh }}\right)$. Here $g\left(\mathbf{R} ; R_{\text {mesh }}\right)$ represents the normalized junction pair distribution function, which maps the probability of finding a second junction as a function of position along a radial axis originating from the first junction. The entropic penalty for bridge formation at distances $\mathbf{R}$ will be large when $g(\mathbf{R})<1$ for $\|\mathbf{R}\|<R_{\text {mesh }}$ such that junctions are locally "depleted" on the length scale of a dangling chain. The penalty becomes even larger when $R_{\text {mesh }}$ is larger than the preferred size (equilibrium end-to-end distance) of the free or dangling chain, in which case a bridged chain is also forced to stretch. If the junction spacing is sufficiently large, it may be thermodynamically favorable for a chain to remain in the dangle state or to form a loop. Because the loop state is intramolecular, it is assumed to be independent of local network geometry, similar to the dangle state.

To incorporate these expectations into the network model, we count bound chain configurations as the sum of bridges and loops, with the bridge configurational integral $\Omega_{B}$ weighted by the local junction density. Thus $Z_{2}=\phi_{j}^{2} \Omega_{B}+\phi_{j} \Omega_{L}$, where $\phi_{j}^{2} \Omega_{B}$ is the conformational entropy of bridged chains, and the corresponding configurational integral is $\Omega_{B}=\int P(\mathbf{R}) g(\mathbf{R}) d \mathbf{R}$. In analogy to the dangle state, the entropy of loops is $\phi_{j} \Omega_{L}$, and we choose to let $\Omega_{L}$ take the same functional form as $\Omega_{f}$, i.e. $\Omega_{L} \sim \int P(\mathbf{R}) d \mathbf{R}$. However, we restrict the bounds of this conformational integral to a small distance $l$ approximating the endto-end distance of looped chains. This integration limit represents a renormalized cubic form of the classical JacobsonStockmayer factor, which quantifies the entropic cost of constraining a polymer chain to a cyclic conformation. ${ }^{40-42}$. In our case, $l$ can be estimated directly from rheological data (see $\mathbf{I n}$ clusion of loops below) or treated as an adjustable parameter in order to generate constrained model "fits" to experimental data. From eq. 1, $K_{2}=\left[\left(\phi_{j} \Omega_{B}+\Omega_{L}\right) / 2 \Omega_{f}\right] \exp (-\beta \Delta E)$.

The preceding theoretical considerations provide the following integral expression for the ratio of $K_{1}$ to $K_{2}$ for a telechelic polymer:

$$
\frac{K_{1}}{K_{2}}=\frac{4 \Omega_{f}}{\Omega_{B}+\Omega_{L} / \phi_{j}}
$$

The ratio is independent of binding energy. It is possible to neglect loops entirely by setting $\Omega_{L}=0$. In this case, the above ratio becomes independent of junction density and is simply:

$$
\frac{K_{1}}{K_{2}}=\frac{K_{1}}{K_{B}}=\frac{4 \Omega_{f}}{\Omega_{B}}=\frac{4 \int P(\mathbf{R}) d \mathbf{R}}{\int P(\mathbf{R}) g(\mathbf{R}) d \mathbf{R}} \geq 4
$$

Taken together, eq. $\mathbf{2}$ and eq. $\mathbf{3}$ distill our key prediction for the reversible binding of telechelic polymers: we expect $K_{1}>4 K_{2}$ in any network with spatially correlated junctions. Although both associative domains have the same intrinsic binding strength (e.g., the enthalpy of association is the same), the ef- 
fective binding strengths of the domains differ. This discrepancy between $K_{1}$ and $K_{2}$ is a consequence of different entropic constraints that govern the two binding events. Whereas translational entropy is lost during both associations, additional conformational entropy is lost in the second association due to restrictions on end-to-end distance enforced by the spatial separation of network junctions. In effect, the network binding affinity of a chain end is reduced whenever the other chain end is already bound. This conformational entropic penalty is expected to decrease as a probe chain increases in size relative to the mesh. For an infinitely long telechelic probe in a finite mesh, the effect of local network structure should disappear completely (apart from topological constraints imposed by the network strands). In this case the untethered chain end would behave like a free chain end: it would have a global "view" of the network (it could access all open sites) such that bridge formation would not be constrained by the location of the other end. $K_{1}=4 K_{2}$ is predicted in this mean-field limit due to the degeneracy of the dangle state. We emphasize that our model is not a self-consistent thermodynamic model for establishing the point of network formation. ${ }^{28,43}$ Rather, we presuppose that the conditions are such that a gel is in fact formed, in order to explore the influence of network structure on the state populations of the chains.

Connection to diffusion mechanisms. The above thermodynamic model predicts the relative magnitudes of $K_{1}$ and $K_{2}$ as a function of the size of a probe chain in relation to the mesh size of the network. Under the assumption that $g(\mathbf{R})$ is a function of $R_{m e s h}$ only, eq. 3 comes to depend on a single parameter, the dimensionless probe size $r \equiv R_{\text {probe }} / R_{\text {mesh }} \sim b_{k} N^{1 / 2} M^{-1 / 3}$. The model can thus be tested by placing increasingly large monodisperse test chains in networks of various sizes and measuring $K_{1}$ and $K_{2}$. These binding constants together specify the equilibrium fraction of free (i.e., hopping) chains as $[f]=\left[1+K_{1}+K_{1} K_{2}\right]^{-1}=\left[1+K_{e f f}^{2}\right]^{-1}$, where $K_{\text {eff }}=\left(K_{1}+K_{1} K_{2}\right)^{1 / 2}$ represents the effective binding coefficient for the entire chain. Below we infer experimental $K_{1}$ and $K_{2}$ values from extensive measurements of polymer self-diffusivities made within telechelic protein hydrogels. The equilibrium constants are obtained under the assumption that hopping represents the only diffusive mode. Interpreting the data in this way, we find that eq. 3 accurately describes the experimental equilibrium constant ratios. This concordance provides indirect evidence for the predominance of hopping in comparison to other diffusive modes (e.g. walking or cluster diffusion) in the networks examined.

Generalization to chains with multiple stickers. The derivation of binding constants for telechelic chains may be generalized to obtain $S$ total equilibrium constants $K_{i}$ that govern the binding to the network of multisticker chains with $i \leq S$ associative domains bound to the network at any given time. The chain is first decomposed into $S-1$ flexible "blocks" between adjacent stickers, $i$ of which are bound. The entropy associated with each block is then calculated, and $Z_{i}$ is obtained as the product of the numbers of configurations available to each block. We derive the exact $K_{i}$ values for the cases of $S=3$ and $S=5$ in the Supporting Information (Table S1). In analogy to telechelic polymers, our key prediction for multisticker chains is that the conformational restrictions enforced by junction separation influence each association, such that $K_{i}>K_{i+1}$ holds for all $i \leq S-1$.

\section{RESULTS AND DISCUSSION}

Network design and characterization. We designed a series of reversible telechelic networks to test the predictions of the model just described. Artificial proteins are well suited to exploring the physical properties of reversible networks: they are structurally well-defined, monodisperse, and easily varied by changing their DNA coding sequences. We cloned and expressed a family of $21 \mathrm{P}\left(\mathrm{E}_{n} \mathrm{P}\right)_{m}$-type protein polymers (Figure $\mathrm{S} 2$ ), where $\mathrm{P}$ is an associative domain that forms pentameric coiled-coils (network junctions), and $\mathrm{E}_{n}$ is a flexible elastin-like linker (Tables S2 and S3). When swollen in aqueous buffer, $\mathrm{PE}_{n} \mathrm{P}$ proteins formed transparent hydrogels with classical "Maxwell-type" rheological signatures (Figures S3 and S4). We could easily vary the mesh size and terminal modulus of each gel by changing the number of repeats of the elastin midblock $(n=3-24)$, without significantly perturbing the network relaxation rate (Figure S5 and Table S4). The terminal network modulus of each " $n$-mesh" approximated the molecular weight dependence expected from rubber elasticity theory $\left(G^{\prime} \sim M^{v}\right)$, with $v=-1$ expected for affine networks, ${ }^{44}$ and $v=-0.9 \pm 0.2$ observed experimentally (Figure S5).

Measurement of equilibrium constants. The equilibrium constants $K_{1}$ and $K_{2}$ for telechelic polymers in each $n$-mesh were estimated by measuring the effective diffusivities of sizematched $(N=M)$ and mismatched $(N \neq M)$ fluorescently-labeled test chains (" $n$-probes", $n=3-48$ ) having either zero $\left(\mathrm{E}_{n}^{*}\right)$, one $\left(\mathrm{E}_{n} \mathrm{P}^{*}\right)$ or two $\left(\mathrm{PE}_{n} \mathrm{P}^{*}\right)$ terminal coils, where $*$ indicates that the proteins have a $\mathrm{C}$-terminal cysteine for fluorophore conjugation (Figure 3A). The effective diffusivities of the probes were measured by fluorescence recovery after photobleaching (FRAP). ${ }^{45-46}$ In this technique, a small region of a sample labeled with a dilute fluorescent species is briefly exposed to a high-intensity laser to promote local, irreversible inactivation of the fluorophore. Subsequent recovery of fluorescence in the photobleached region ("bleach spot") reports on the mobility of the labeled species. The effective diffusivity $D_{S}$ of a test chain carrying $S$ associative domains is obtained by fitting the FRAP trace to a renormalized Fickian diffusion model (see Supporting Information). ${ }^{25,45-46}$ The diffusivity can be related to binding equilibrium constants through the equation:

$$
\frac{D_{0}}{D_{S}}=1+K_{1}+K_{1} K_{2}+\cdots+\prod_{i=1}^{S} K_{i}=1+\sum_{j=1}^{S}\left(\prod_{i=1}^{j} K_{i}\right)
$$

where $D_{0}=D_{f}$ is the effective Fickian diffusivity of the test chain in the free state.

The derivation of eq. $\mathbf{4}$ assumes that reversible binding of the chains is fast relative to the time scale of free diffusion during the FRAP experiment, which we validated previously for $\mathrm{PE}_{n} \mathrm{P}$ gels. ${ }^{25}$ For the experimental regime probed here, we estimate that $k_{\text {on }}{ }^{*} a^{2} / D_{0} \approx 10^{2}-10^{3}$ for the case when the bleach spot radius $a=10 \mu \mathrm{m}$, and $k_{o n}{ }^{*}$ is the (concentration-dependent) pseudo-first-order association rate constant. Eq. 4 further assumes that network chains have a negligible mobility once bound, such that all chain migration occurs through the hopping mechanism, i.e., single chains must completely disengage from the network (enter the free state) in order to undergo center-of-mass translation. Therefore, if the equilibrium constants inferred from this equation are consistent with our thermodynamic model, hopping is indicated to be the dominant diffusive mode. Inversely, if the correspondence to theory is poor, other 
A

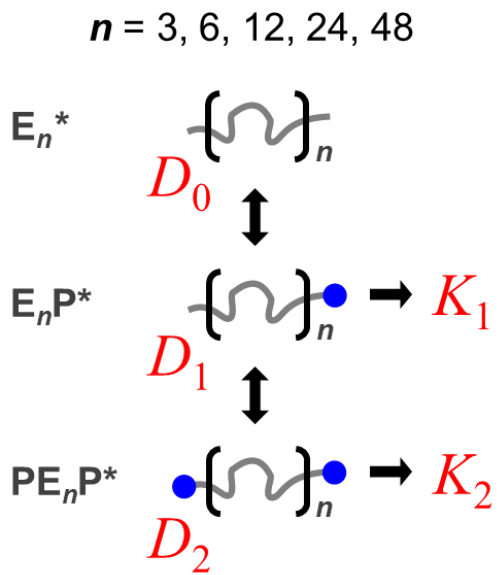

C

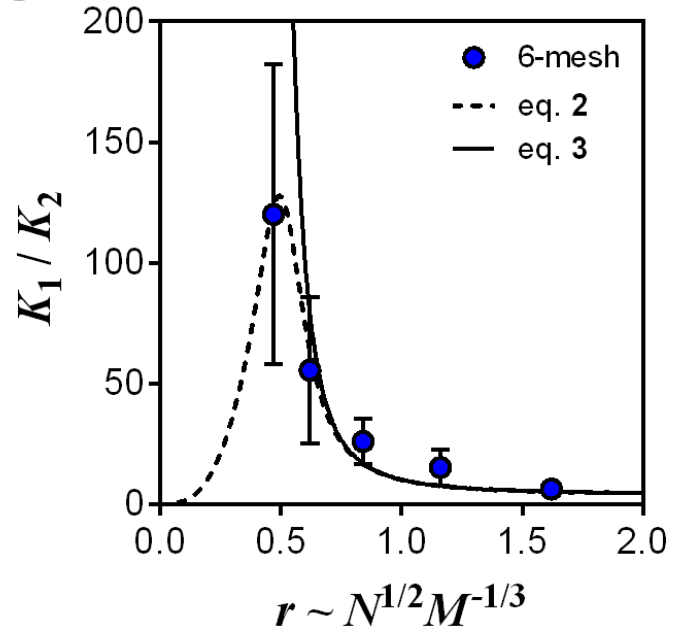

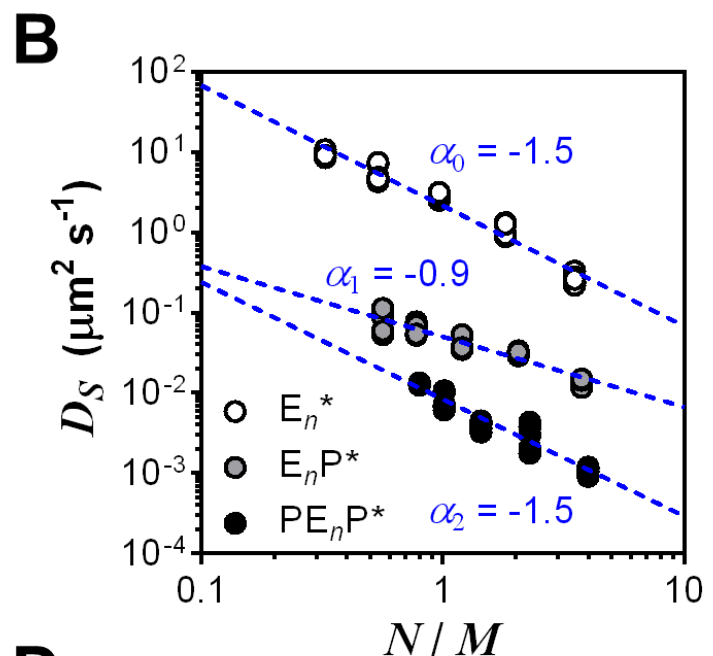

D

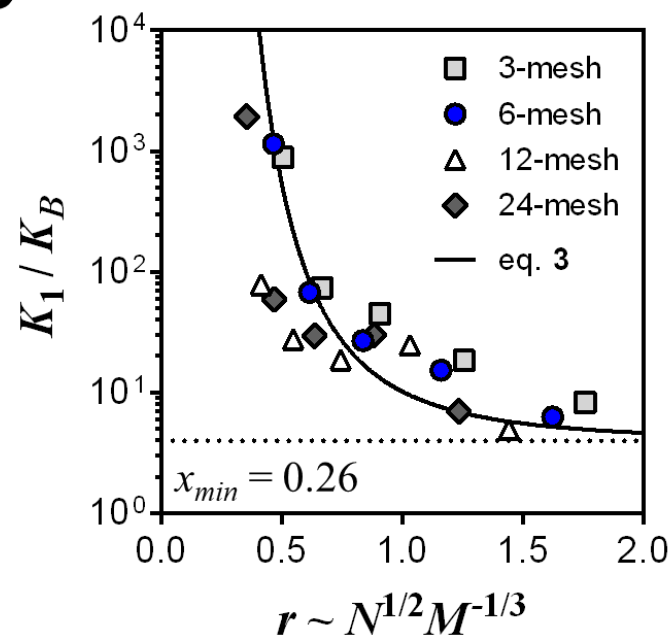

Figure 3. Experimental binding constant ratios inferred from diffusion measurements: correspondence to theory supports chain hopping. (A) Elastin-like probes with different lengths and numbers of stickers were placed in size-matched $(N=M)$ and mismatched $(N \neq M)$ " $n$-mesh" $\mathrm{PE}_{n} \mathrm{P}$ networks. Equilibrium constants were inferred from FRAP diffusivity measurements via eq. 4, i.e. assuming exclusive hopping. Estimating a single $K_{1} / K_{2}$ ratio requires independent diffusivity measurements from three different probe types having zero (E $\left.{ }_{n}^{*}\right)$, one $\left(\mathrm{E}_{n} \mathrm{P}^{*}\right)$ or two $\left(\mathrm{PE}_{n} \mathrm{P}^{*}\right)$ stickers. (B) Representative diffusivities in a 6-mesh (80 total measurements, $\geq 4$ per probe type). Approximate molecular weight exponents for each probe type were estimated from coarse power-law fits (see discussion in main text). (C) The experimental $K_{1} / K_{2}$ data (mean \pm std. dev.) in the 6-mesh reveal binding asymmetry for telechelic chains. The data are plotted against the dimensionless probe size $r \equiv R_{\text {probe }} / R_{\text {mesh }} \sim b_{k} N^{1 / 2} M^{-1 / 3}$. The "loop-free" prediction from eq. 3, and the single-parameter fit of eq. 2 with $x_{\min }=0.26$ are shown; $x_{\min }=l_{\min } / c$ is the best-fit dimensionless integration limit for the looping configurational $\Omega_{L}$ (see discussion in main text). (D) Loop subtraction with $x_{\min }=0.26$ permits comparison of the $K_{1} / K_{2}$ data from each mesh to eq. 3 (shown are mean $K_{1} / K_{\mathrm{B}}$ ratios deduced from 298 total measurements, $\geq 4$ per probe type). Error bars in panel D are omitted for clarity, but are analogous in size to those in panel C. The dotted line represents the mean-field limit $K_{1} / K_{B}=4$.

diffusive modes must be invoked. Similar logic is readily extended to multisticker chains (see Evidence for multisticker chain hopping below).

To begin, we obtain $D_{0}$ by monitoring the fluorescence recovery rate of an $\mathrm{E}_{n}{ }^{*}$ test chain that cannot bind the network and is therefore "locked" in the free state. $K_{1}$ is then obtained by measuring the mobility $D_{1}$ of an $\mathrm{E}_{n} \mathrm{P}^{*}$ probe, and entering the values of $D_{0}$ and $D_{1}$ into eq. 4 . Note that the equilibrium constant thus obtained reflects the association strength of chains with only one sticker $\left(\mathrm{E}_{n} \mathrm{P}^{*}\right.$ probes $)$, i.e. $K_{1}=\phi_{j} \exp (-\beta \Delta E)$. Because the dangle state is degenerate for telechelic chains, the equilibrium constant measured for $\mathrm{E}_{n} \mathrm{P}^{*}$ must be multiplied by two to obtain $K_{1}$ for a $\mathrm{PE}_{n} \mathrm{P}^{*}$ probe. Finally, $K_{2}$ for telechelic chains is obtained by measuring $D_{2}$ for a $\mathrm{PE}_{n} \mathrm{P}^{*}$ probe, and supplying it into eq. $\mathbf{4}$, together with $D_{0}$ and $K_{1}$. At each step we allow $D_{0}$ to decrease following a default Rouse scaling $D_{0} \sim N^{-1}$, to take into account the added friction of the additional $\mathrm{P}$ domain. Although the conformational properties of this domain in the unbound state are unknown, adjusting $D_{0} \sim N^{v}$ using a weaker $(v=-0.6)$ or stronger $(v=-2)$ scaling exponent has essentially no effect on the inferred binding constant ratios, i.e. this scaling adjustment is minor. Iterating this procedure for many probe-mesh combinations provides experimental $K_{1} / K_{2}$ ratios as a function of $N$ and $M$, which are proportional to the molecular weights of the test chain and the network, respectively. 
The FRAP method, combined with the total control of chain structure characteristic of protein synthesis, allowed us to tune the properties of the network independent of the embedded fluorescent tracers and provided great flexibility in testing our model predictions. We acquired a total of 298 FRAP traces on 15 different probes in 4 different meshes (60 unique probe-mesh combinations) in order to explore a wide range of different probe-to-mesh ratios $\left(r=R_{\text {probe }} / R_{\text {mesh }}\right)$. Since the shape and intensity of the bleach spot were found to be moderately sensitive to the structure of the probe (Figure S6), we acquired an additional 173 control FRAP traces on size-matched $\mathrm{E}_{n}{ }^{*}$ probes in each of the 4 meshes. Using a generalized Gaussian bleach spot fit-and-track algorithm, we found the measured diffusivity to be insensitive to variation in the bleach spot profile over a wide range of bleach efficiencies. Representative FRAP traces for each of the probes in the 6-mesh network, along with their corresponding Fickian diffusion fits, are presented in Figure S8. The values of $D_{S}$ obtained from all 60 probe-mesh combinations ( $n \geq 4$ replicates per combination) are plotted in Figure S9.

Power-law fits to diffusivity data. Our model predicts that, as probe chains increase in size above the mesh size of the network, $K_{1} / K_{2}$ will decrease monotonically. Coarse power-law fits to the diffusivity dataset provide a qualitative test of this prediction. Polymer diffusivities typically exhibit power-law scaling with molecular weight, i.e. $D \sim N^{\alpha}$ where the molecular weight exponent $\alpha$ is negative (e.g. $\alpha=-1$ in the Rouse model). ${ }^{15,47-48}$ By measuring these exponents, we can infer additional molecular weight exponents $\beta$ for each equilibrium constant $K_{i} \sim N^{\beta}$ from a scaling analysis of eq. 4. In the 6-mesh, $\alpha_{0}=-1.5, \alpha_{1}=-0.9$ and $\alpha_{2}=-1.5$ for $D_{0}, D_{1}$ and $D_{2}$ respectively (Figure 3B). From eq. 4 we infer $\beta_{1}=\alpha_{0}-\alpha_{1}=-0.6$ for $K_{1}$ as well as $\beta_{2}=\alpha_{1}-\alpha_{2}=0.6$ for $K_{2}$. Critically, the molecular weight exponent for $K_{2}$ is positive, implying increasing association strength of the second chain end as the test chain becomes longer. Moreover, the exponent corresponding to the $K_{1} / K_{2}$ ratio, $\beta_{1}-\beta_{2}=-1.2$, is strongly negative, consistent with weakening of the entropic constraint for bridge entry as the chain length grows. These results are in qualitative agreement with our model.

That the free-chain diffusivity exhibits a molecular-weight exponent that is stronger than Rouse-like $\left(\alpha_{0}=-1.5\right.$ for $\left.D_{0}\right)$ may indicate crossover between different regimes (i.e., Rouse to reptation-like $\left.{ }^{49}\right)$ as the probe chains become large relative to the mesh size; its precise origin requires further investigation. We attribute the negative value of $\beta_{1}$ to excluded volume effects (e.g., end group association on the test chain starts to become sterically hindered by the large pervaded volume of the chain itself), which are not included in our model. Presumably such effects would hinder both associations to a similar extent, in which case they should minimally affect the experimental $K_{1} / K_{2}$ ratio. Note that the binding constants are not expected to have formal power-law dependencies on $N$, so there is no rigorous basis to expect the molecular weight exponents $\beta$ to hold generally.

Quantitative comparison to theory. A quantitative comparison of the experimental equilibrium constant ratios to the thermodynamic model (eq. 2 and eq. 3) requires estimates of the mesh size $R_{\text {mesh }}$ and the probe size $R_{\text {probe }}$. Reasonable estimates for these key parameters are readily obtained (see Supporting Information)..$^{50-53}$ The estimated probe sizes range from 6.1 to $21.4 \mathrm{~nm}$, whereas the smallest (3-mesh) and largest (24-mesh) networks have mesh sizes of $12.2 \mathrm{~nm}$ and $17.3 \mathrm{~nm}$, respectively (Table S4 and Table S5). Since we examine chain lengths below the thermal blob size (such that excluded volume interactions and correlations can be largely neglected; see Supplementary Equations and Derivations in the Supporting Information), we evaluate the configurational integrals using the formula $P\left(\mathbf{R} ; N, b_{k}\right)=\left(3 / 2 \pi N b_{k}{ }^{2}\right)^{3 / 2} \exp \left(-3 \mathbf{R}^{2} / 2 N b_{k}{ }^{2}\right),{ }^{44}$ the probability density function for an ideal (Gaussian) chain. For simplicity, we approximate $g\left(\mathbf{R} ; R_{m e s h}\right)$ as a Heaviside step function activated at $R_{m e s h}$. Except $\Omega_{L}$, all configurational integrals are taken over the range of possible sizes of the chain, which for a Gaussian chain is the interval $[0, \infty]$.

Figure $3 \mathrm{C}$ plots $K_{1} / K_{2}$ predicted by eq. 3 as function of the reduced (dimensionless) probe size $r$, together with experimental $K_{1} / K_{2}$ data obtained from FRAP experiments in the 6mesh. The experimental $K_{1} / K_{2}$ ratio decays monotonically as the probe size increases, in good agreement with the prediction from eq. 3 (no loops). This correspondence is obtained without any externally fit model parameters or data transformation, beyond obtaining reasonable estimates for chain and network dimensions. Note that the difference in binding constants is significant even when the probe chains have the same dimensions as the mesh $\left(K_{1} / K_{2} \approx 20\right.$ is observed for $\left.r=1\right)$, pointing to the critical role played by local junction order as opposed to chain stretching. The data thus support a central hypothesis of the thermodynamic model: chains in the bridge state are conformationally constrained beyond the mean-field prediction, and the conformational constraint reduces the effective association strength of the chains. The entropic penalty for bridge formation increases as the chains decrease in size within a network of fixed dimensions.

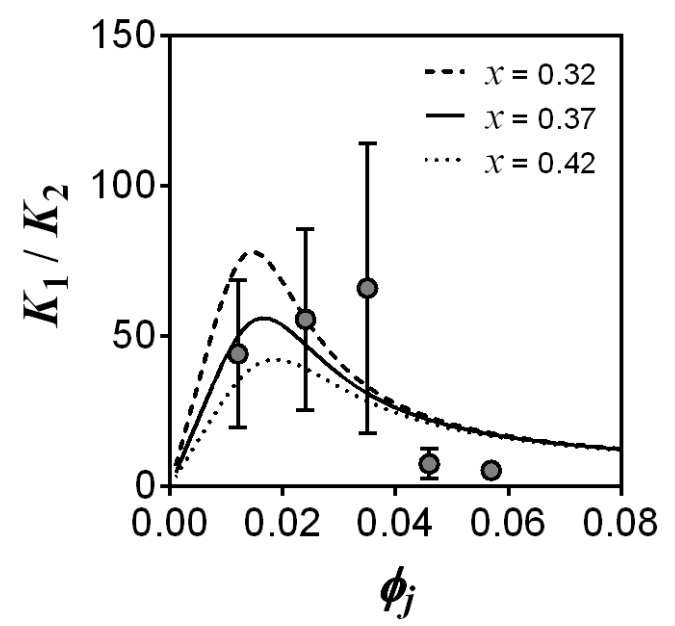

Figure 4. Concentration dependence of the binding constant ratio in a 6-mesh network. $K_{1} / K_{2}$ is proportional to $\phi_{j}$ (junction density) for small $\phi_{j}$, then falls sharply above $\phi_{j} \approx 0.04$ due to a decrease in junction spacing. Eq. 2 qualitatively captures this behavior, with $x_{\text {min }}=0.37 \pm 0.05, b=0.95 \mathrm{~nm}$ and $N=72$. Error bars depict mean \pm std. deviation from $n=76$ total measurements, with $\geq 2$ measurements per probe type per concentration. The mass concentrations range from $5-25 \%(w / v)$, i.e., $\rho=50-250 \mathrm{mg} / \mathrm{mL}$.

This finding has important implications for the dominant mechanism of chain diffusion. Reducing the effective association strength of the chains increases the fraction of free (dissociated) chains. Moreover, using eq. 4 to infer experimental binding constants implicitly assumes negligible translational motion in the bound state (i.e., all fluorescence recovery is at- 
tributed to the movement of free chains). The fact that this inference provides binding constant ratios consistent with eq. 3 supports the assumption that at equilibrium, effective diffusion in $\mathrm{PE}_{n} \mathrm{P}$ networks is dominated by hopping. For this assumption to be invalidated, an alternative diffusive mode having the same functional dependence on network structure as the equilibrium fraction of free chains would need to be identified. Other diffusive modes such as walking ${ }^{27}$ or multichain diffusion ${ }^{15}$ may still be important for related dynamic network phenomenon (e.g. stress relaxation).

Inclusion of loops. Dimensional analysis of eq. 3 reveals that, when loops are neglected and $g(\mathbf{R})$ is the unit step function, $K_{1} / K_{2}$ is determined solely by the dimensionless probe size $r$. In eq. 2, however, loop inclusion introduces a concentration dependence via the term $\Omega_{L} / \phi_{j}$. Careful analysis of this concentration-dependence further substantiates the thermodynamic model. The concentration may be expressed as $\phi_{j}=\left(2 c / R_{\text {mesh }}\right)^{3}$, where $c=1.89 \mathrm{~nm}$ represents the "cluster size" or characteristic dimension of a network junction (see Supporting Information for the numerical determination of this parameter). Since $\Omega_{L}$ is integrated over the interval $[0, l]$, it is convenient to define a second dimensionless parameter $x \equiv l / c$ and integrate over the new interval $[0, x]$. In this case the ratio $\Omega_{L} / \phi_{j} \sim(l / c)^{3}$ is approximately independent of $c$ for fixed values of $x$ (Figure S10). With this construction, eq. 2 depends solely on $r$ and $x$, and accounting for loops amounts to obtaining a reasonable estimate for the dimensionless integration limit $x$.

Since $\Omega_{L}$ and $\Omega_{B}$ are theoretically related to the gel modulus (to first order, only bridged chains are elastically effective), it is possible to estimate $x$ based on rheological data (eq. S10 and Figure S11). This provides $x=x_{\text {rheo }}=0.41 \pm 0.28$ across the four different meshes examined here (Table S4). In practice, eq. 2 is quite sensitive to the value of $x$ due to the cubic nature of the volume integral (i.e., $\Omega_{L} / \phi_{j} \sim x^{3}$ ). One can also obtain $x=x_{\text {min }}$ by systematically varying $x$ in order to minimize residuals between model-constrained "fits" of eq. 2 to the experimental $K_{1} / K_{2}$ values (Figures S11 and S12), which provides. Figure $3 \mathrm{C}$ presents a single-parameter fit of eq. 2 to the 6-mesh data with $x_{\min }=0.26\left(x_{\min }=0.32 \pm 0.09\right.$ is obtained from collating the estimates across each of the four meshes, Table S4). The fit captures an observed softening of the experimental rise in $K_{1} /$ $K_{2}$ at low $r$ (relative to the "loop-free" prediction of eq. 3 ), an effect attributable to the increased prevalence of loops. The fits are qualitatively better in the smaller (3- and 6-mesh) gels, which we attribute to decreased local order in the more open (12- and 24-mesh) gels, for which $K_{1} / K_{2}$ also tends to be lower (Figure S12).

Although the data in each mesh were acquired at fixed mass concentrations $(\rho=100 \mathrm{mg} / \mathrm{mL})$, the junction density $\phi_{j}$ is different in each mesh because of differences in midblock length. Accounting for loops provides a means to compare the measurements of $K_{1} / K_{2}$ from each mesh (Figure S9). This comparison is obtained by subtracting the concentration-dependent terms (i.e., $\Omega_{L} / \phi_{j}$ ) from the experimental $K_{1} / K_{2}$ ratios to obtain the "loop-free" ratio $K_{1} / K_{\mathrm{B}}$. Combining eq. 2 and eq. 3 reveals that $K_{1} / K_{B}=\left[K_{2} / K_{1}-\Omega_{L} / 4 \phi_{j}\right]^{-1}$. Using $x_{\min }=0.26$ to perform the subtraction leads to a satisfying collapse of the entire dataset (298 total measurements) onto the universal "loop-free" curve of eq. 3 (Figure 3D). The $K_{1} / K_{B}$ values obtained in this way vary over a remarkable 300 -fold range, exceeding $10^{3}$ for $r=0.36$, obtained with the (smallest) 3-probe in the (largest) 24-mesh. Moreover, the data appear to approach the predicted mean-field asymptote: $K_{1} / K_{B}=4.9-8.3$ is obtained from the largest probes in each mesh over the domain $r=1.2-1.8$. The coherence of this large dataset with model predictions further reinforces the validity of the hopping inference.

Concentration dependence. In order to test the concentration dependence of eq. 2 more directly, we acquired an additional 54 FRAP traces in size-matched $(N=M)$ 6-mesh networks at 4 additional mass concentrations, varying $\rho$ between 50 and $250 \mathrm{mg} / \mathrm{mL}$ (equivalent to $\phi_{j}=0.012-0.057$, all above the gelation point). The binding constants obtained from these measurements are plotted in Figure 4, along with eq. 2 evaluated at $x_{\text {min }}=0.37 \pm 0.05$ (optimized for the concentration dataset) Substantial formation of loops is evident experimentally at the lowest concentrations, with $K_{1} / K_{2} \sim \phi_{j}$ for small $\phi_{j} . K_{1} / K_{2}$ then falls sharply above $\phi_{j}=0.035$, consistent with increased bridge formation as the junction spacing becomes smaller (with a fixed probe size). Although the experimental decrease in $K_{1} / K_{2}$ is sharper than eq. 2 predicts, the model qualitatively captures a local maximum in the data, which corresponds to a crossover between loop-dominant (low $r$ ) and bridge-dominant (high $r$ ) regimes. Excluded volume effects not included in the model are likely to be important in the real network at higher concentrations, and could shift the location of this predicted local maximum.

It is interesting to note that eq. $\mathbf{2}$ specifies a region at very low $\phi_{j}<\phi_{j}{ }^{*}=\Omega_{L} /\left(4-\Omega_{B}\right)$, for which $K_{1}<K_{2}$ and the difference in binding strengths inverts. This concentration regime lies below the theoretical mean-field percolation threshold for a pentameric network, $p_{c}=1 /(f-1)=1 / 4$ for $f=5 .{ }^{44}$ Substituting $\phi_{j}{ }^{*}$ into eq. S10 provides the theoretical bridge fraction at this concentration, $[B]^{*}=\Omega_{B} / 4 \leq p_{c}$, i.e. this concentration is so low that the network no longer exists. Hence we expect $K_{1}>K_{2}$ for telechelic chains whenever a network is present, at least within networks assembled from pentameric crosslinking domains.

Evidence for multisticker chain hopping. The presence of additional associative domains on a polymer chain increases its binding to the network. As a result, a multisticker chain with $S$ $\geq 2$ stickers is intrinsically less likely to hop than the corresponding telechelic polymer. However, the chain will also experience additional conformational restrictions whenever two or more of its stickers form a bridge between spatially separated junctions. The entropic penalty for "full" association of a multisticker chain (i.e., all stickers in the bridge state) may thus be very high, such that binding is substantially reduced. In analogy to telechelic polymers, we can define an effective network binding coefficient $K_{\text {eff }}$ for multisticker chains as:

$$
K_{\text {eff }}=\left[\sum_{j=1}^{S}\left(\prod_{i=1}^{j} K_{i}\right)\right]^{1 / S}
$$

such that $D_{0} / D_{S}=1+K_{\text {eff }}^{S}$ (cf. eq. 4). For telechelic polymers, the effect of local network structure is to reduce $K_{2}$ relative to $K_{1}$, such that $K_{\text {eff }}=\left(K_{1}+K_{1} K_{2}\right)^{1 / 2}$ is smaller than expected. Similarly for a multisticker chain, the effective binding strength of a given sticker is attenuated by the presence of other concurrently bound stickers on the same chain such that $K_{i+1}$ is always less than $K_{i}$, thereby reducing $K_{\text {eff }}$ even further (relative to the case of the same polymer in an uncorrelated or mean-field network). As with telechelics, this should amplify the fraction of free chains, and thus the probability that a chain will migrate by "hopping" (complete network disengagement before rebinding) (Figure 1). Comparing the predicted binding strengths and diffusivities of multisticker chains with experiments thus provides 
a stringent test of the effect of local network structure on equilibrium chain transport.
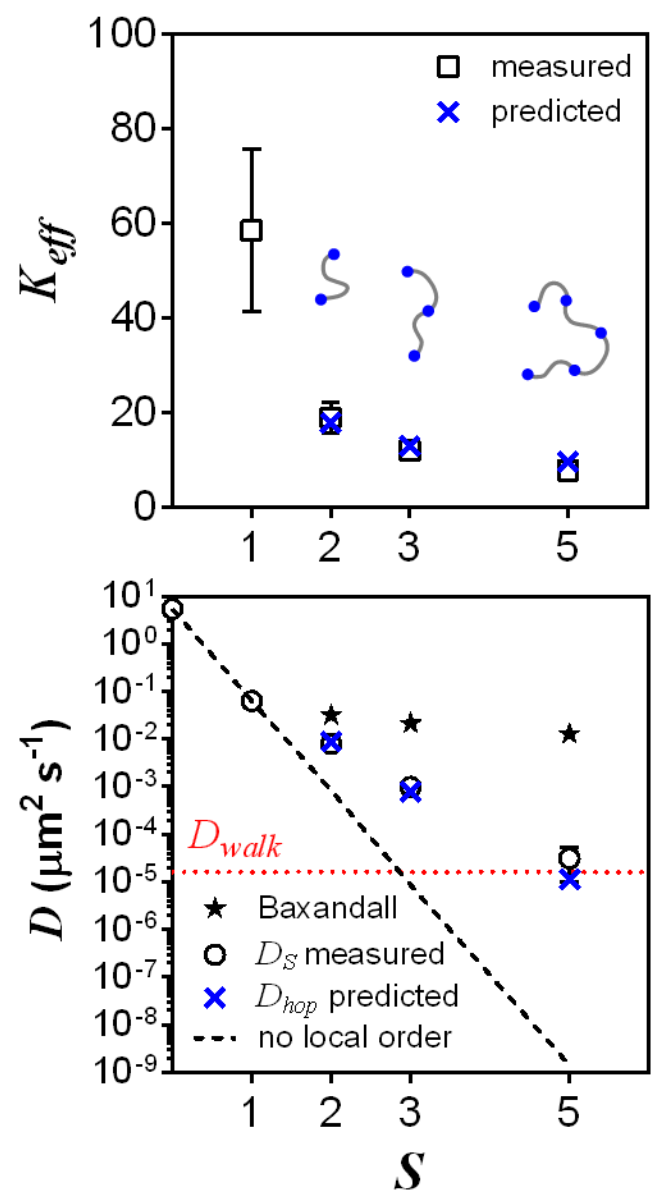

Figure 5. Hopping dominates the diffusion of multisticker chains in telechelic networks. (Top) Measurements of $K_{\text {eff }}$ inferred from experimental $D_{S}$ and eqs. $\mathbf{4}$ and $\mathbf{5}$ are in good agreement with model predictions for chains with $S=2,3$ and 5 associative domains. (Bottom) Comparison of $D_{S}$ measured by FRAP and $D_{\text {hop }}$ predicted from the thermodynamic model. All measurements were taken in $10 \%(w / v) 6$-mesh networks using size-matched probes (i.e., the intersticker spacing $N$ corresponds to the mesh size $M$ ). Symbols depict mean \pm std. deviation from $n \geq 4$ measurements per probe type. All model predictions were made using $x_{\min }=0.26$. The prediction from Baxandall ${ }^{27}$ assumes $D_{S} \sim S^{-1}$. "No local order" assumes that all binding events have the same strength as the first association, i.e. all $K_{i}=K_{1}$. The theoretical mobility of bound chains is calculated as $D_{\text {walk }}=R_{\text {mesh }}{ }^{2} / 6 \tau_{b}=1.61 \times 10^{-5} \mu \mathrm{m}^{2} \mathrm{~s}^{-1}$.

We synthesized multisticker probes with $S=3$ and $S=5$ stickers, and compared their effective binding strengths in a telechelic 6-mesh network to the exact theoretical predictions for polymers of this type (see Supporting Information for a derivation of the key equations; representative binding configurations for a chain with $S=3$ stickers are presented in Figure S1; relative theoretical values of each $K_{i}$ for $S=5$ are presented in Table S1). To predict $K_{\text {eff }}$ for multiblocks, the model requires an estimate for $K_{1}$, obtained by FRAP using the $\mathrm{E}_{n}{ }^{*}(S=0)$ and $\mathrm{E}_{n} \mathrm{P}^{*}(S=1)$ probes. To correctly account for loops, we also integrate each loop configurational with $x_{\min }=0.26$ based on the minimization of residuals for telechelic chains in the 6-mesh (see the eq. 2 fit in Figure 3C). The prediction for $S=2$ thus contains some information from a model-constrained fit to data from the same probe type, whereas the predictions for $S=3$ and $S=5$ do not. We observe striking agreement between the new multisticker predictions and the experimental values of $K_{\text {eff }}$ inferred from eqs. 4 and 5 (Figure 5).

In analogy to telechelics, the fraction of free multisticker chains is readily computed as $[f]=\left[1+K_{\text {eff }}{ }^{S}\right]^{-1}$, providing a simple way to estimate the contribution of hopping to the total diffusivity. Assuming the measured diffusivity $D_{S}$ contains contributions from only hopping and walking, then $D_{S}=D_{\text {hop }}+D_{\text {walk }}=[f] D_{0}+R_{\text {mesh }}^{2} / 6 \tau_{b}$. Here we have assumed that a bound chain can translate of order the mesh size within a bound time $\tau_{b}=\omega_{c}{ }^{-1} \approx 1 \mathrm{sec}$, set by the relaxation rate of the network (Figure S5). Using the theoretical $[f]$ and experimental $D_{0}$, we can directly predict $D_{h o p}=[f] D_{0}$ with the model. If this prediction is similar to $D_{S}$ as measured by FRAP, we can infer that hopping is the dominant mode of chain migration.

Figure 5 plots $D_{S}$ measured by FRAP together with the hopping prediction $D_{\text {hop }}$ for all multistickers. The hopping prediction is essentially exact for $S=2$ and $S=3$. Remarkably, $D_{\text {hop }} / D_{S}=0.36$ for $S=5$, suggesting that ca. $36 \%$ of these multisticker chains migrate by hopping, whereas the remaining chains undergo translational motion through a new diffusive mechanism. The theoretical bound mobility of telechelic chains in the host network is $D_{\text {walk }}=R_{\text {mesh }}{ }^{2} / 6 \tau_{b}=1.61 \times 10^{-5} \mu \mathrm{m}^{2} \mathrm{~s}^{-1}$ (calculated for a 6-mesh). Guest chains with $S=5$ stickers appear to approach this lower bound (Figure 5B), suggesting that the new diffusive mode represents reorganization of the host network itself (i.e., "walking" of telechelic chains) around the multisticker guest. In support of this claim, we observe that $\left(D_{\text {hop }}+D_{\text {walk }}\right) / D_{S}=0.88 \approx 1$ for these guest chains. The fact that $D_{S} \gg D_{\text {walk }}$ holds for all but the stickiest chain is direct, model-independent evidence that walking and other diffusive modes based on bound configurations (including multichain cluster diffusion) do not contribute meaningfully to the diffusivity of any of the smaller chains.

Most notably, ignoring the influence of local network structure on effective network binding affinity leads to incorrect predictions for $D_{h o p}$ due to a drastic underestimation of the fraction of free chains. For chains with $S=5$ stickers, for example, $[f]=\left[1+\left(K_{1}\right)^{5}\right]^{-1} \approx 1 \times 10^{-9}$ for the case where all stickers bind with the same affinity as the first. Considering local network structure, however, provides $[f]=\left[1+\left(K_{\text {eff }}\right)^{5}\right]^{-1} \approx 1 \times 10^{-5}$, i.e. the number of free chains increases by a factor of $10^{4}$. This result highlights the profound extent to which entropic constraints can influence the dynamics of single chains in reversible networks. Our new dataset also provides a quantitative test of an original mean-field prediction from Baxandall, which also neglects the local structure of the network. ${ }^{27}$ His prediction that multisticker self-diffusion should be Rouse-like, with $D_{S} \sim S^{-1}$, appears too weak. It is possible that this prediction could still hold in the regime of total binding (no free chains). Such a regime is perhaps experimentally accessible for $S \gg 5$.

\section{CONCLUSION}

Several distinct mechanisms of chain diffusion in unentangled associative polymer networks have been proposed over the past three decades. Here we present evidence that hopping (diffusion of "free" chains that are transiently disengaged from the network), can be the dominant mode of chain transport in such systems, even in networks in which the intrinsic association strength is high. We propose a simple thermodynamic origin for this behavior: an entropic penalty that reduces the effective 
strength for all subsequent binding events once the first "sticker" is bound. This effect amplifies the fraction of free chains and promotes hopping, even for chains with many stickers. Predictions of the thermodynamic model explain the observed dependences of the effective diffusion coefficient on chain length (including mismatches in the lengths of network and probe chains), network junction density, and number of stickers in networks formed from monodisperse artificial proteins. We believe the behavior described here to be characteristic of associative macromolecular networks of many different kinds.

\section{ASSOCIATED CONTENT}

\section{Supporting Information}

Contains detailed experimental protocols, additional mathematical derivations, and other supplementary information. This material is available free of charge via the Internet at http://pubs.acs.org.

\section{AUTHOR INFORMATION}

\section{Corresponding Author}

*tirrell@caltech.edu

\section{Present Addresses}

Chemistry Department, Yale University, 225 Prospect St., New Haven, CT 06511

\section{Author Contributions}

\$These authors contributed equally to this work.

\section{Funding Sources}

This work was supported by grant number DMR-1506483 from the Biomaterials Program of the U. S. National Science Foundation. A.K.O. acknowledges support from the National Science Foundation Graduate Research Fellowship under Grant No. DGE-1144469 and an HHMI Gilliam Fellowship. B.R.S. acknowledges support from NIH pre-doctoral training grant 1T32GM112592 and from the Rosen Center for Bioengineering.

\section{ACKNOWLEDGMENT}

We thank Andres Collazo of the Caltech Beckman Imaging Facility for expert assistance with acquisition of FRAP data.

\section{ABBREVIATIONS}

FRAP, fluorescence recovery after photobleaching

\section{REFERENCES}

1. Guo, M.; Pitet, L. M.; Wyss, H. M.; Vos, M.; Dankers, P. Y. W.; Meijer, E. W., J. Am. Chem. Soc. 2014, 136 (19), 6969-6977.

2. Cordier, P.; Tournilhac, F.; Soulie-Ziakovic, C.; Leibler, L., Nature 2008, 451 (7181), 977-980.

3. Zhou, Z.; Yan, X.; Cook, T. R.; Saha, M. L.; Stang, P. J., J. Am. Chem. Soc. 2016, 138 (3), 806-809.

4. Herbst, F.; Seiffert, S.; Binder, W. H., Polym. Chem. 2012, 3 (11), 3084-3092.

5. Yount, W. C.; Juwarker, H.; Craig, S. L., J. Am. Chem. Soc. 2003, 125 (50), 15302-15303.

6. Yount, W. C.; Loveless, D. M.; Craig, S. L., J. Am. Chem. Soc. 2005, 127 (41), 14488-14496.

7. Xu, D.; Craig, S. L., Macromolecules 2011, 44 (13), 5465-5472.

8. Grindy, S. C.; Learsch, R.; Mozhdehi, D.; Cheng, J.; Barrett, D. G.; Guan, Z.; Messersmith, P. B.; Holten-Andersen, N., Nat. Mater. 2015, 14 (12), 1210-1216.
9. Holten-Andersen, N.; Harrington, M. J.; Birkedal, H.; Lee, B. P.; Messersmith, P. B.; Lee, K. Y.; Waite, J. H., Proc. Natl. Acad. Sci. 2011, 108 (7), 2651-2655.

10. Tang, S.; Habicht, A.; Li, S.; Seiffert, S.; Olsen, B. D., Macromolecules 2016, 49 (15), 5599-5608.

11. Annable, T.; Buscall, R.; Ettelaie, R.; Whittlestone, D., J. Rheol. 1993, 37 (4), 695-726.

12. Petka, W. A.; Harden, J. L.; McGrath, K. P.; Wirtz, D.; Tirrell, D. A., Science 1998, 281 (5375), 389-392.

13. Tang, S. C.; Wang, M. Z.; Olsen, B. D., J. Am. Chem. Soc. 2015, 137 (11), 3946-3957.

14. Olsen, B. D.; Kornfield, J. A.; Tirrell, D. A., Macromolecules 2010, 43 (21), 9094-9099.

15. Rubinstein, M.; Semenov, A. N., Macromolecules 1998, 31 (4), 1386-1397.

16. Lutolf, M. P.; Hubbell, J. A., Nat. Biotechnol. 2005, 23 (1), 4755.

17. McKinnon, D. D.; Domaille, D. W.; Cha, J. N.; Anseth, K. S., Adv. Mater. 2014, 26 (6), 865-872.

18. Li, J.; Li, X.; Ni, X. P.; Wang, X.; Li, H. Z.; Leong, K. W., Biomaterials 2006, 27 (22), 4132-4140.

19. Wang, C.; Wu, H.; Chen, Z.; McDowell, M. T.; Cui, Y.; Bao, Z., Nat. Chem. 2013, 5 (12), 1042-1048.

20. Tee, B. C. K.; Wang, C.; Allen, R.; Bao, Z., Nat. Nanotechnol. 2012, 7, 825-832.

21. Timney, B. L.; Raveh, B.; Mironska, R.; Trivedi, J. M.; Kim, S. J.; Russel, D.; Wente, S. R.; Sali, A.; Rout, M. P., J. Cell Biol. 2016, 215 (1), 57-76.

22. Kim, M.; Chen, W. G.; Kang, J. W.; Glassman, M. J.; Ribbeck, K.; Olsen, B. D., Adv. Mater. 2015, 27 (28), 4207-4212.

23. Martino, M. M.; Briquez, P. S.; Guc, E.; Tortelli, F.; Kilarski, W. W.; Metzger, S.; Rice, J. J.; Kuhn, G. A.; Muller, R.; Swartz, M. A.; Hubbell, J. A., Science 2014, 343 (6173), 885-888.

24. Chen, Q.; Tudryn, G. J.; Colby, R. H., J. Rheol. 2013, 57 (5), 1441-1462.

25. Rapp, P. B.; Omar, A. K.; Shen, J. J.; Buck, M. E.; Wang, Z.-G.; Tirrell, D. A., J. Am. Chem. Soc. 2017, 139 (10), 3796-3804.

26. Shen, W.; Kornfield, J. A.; Tirrell, D. A., Macromolecules 2007, 40 (3), 689-692.

27. Baxandall, L. G., Macromolecules 1989, 22 (4), 1982-1988.

28. Semenov, A. N.; Rubinstein, M., Macromolecules 1998, 31 (4), 1373-1385.

29. Green, M. S.; Tobolsky, A. V., J. Chem. Phys. 1946, 14 (2), 80 92.

30. Tanaka, F.; Edwards, S. F., Macromolecules 1992, 25 (5), 15161523.

31. Tripathi, A.; Tam, K. C.; McKinley, G. H., Macromolecules 2006, 39 (5), 1981-1999.

32. Ramirez, J.; Dursch, T. J.; Olsen, B. D., Macromolecules 2018, 51 (7), 2517-2525.

33. Lodge, T. P.; Rotstein, N. A.; Prager, S., In Advances in Chemical Physics, 1990; Vol. 79, pp 1-132.

34. Lodge, T. P., Phys. Rev. Lett. 1999, 83 (16), 3218-3221.

35. McLeish, T. C. B., Adv. Phys. 2002, 51 (6), 1379-1527.

36. Doi, M.; Edwards, S. F., The Theory of Polymer Dynamics. Oxford University Press: 1986.

37. Omar, A. K.; Wang, Z. G., Phys. Rev. Lett. 2017, 119 (11), 117801

38. Hill, T. L., An Introduction to Statistical Thermodynamics. Dover Publications: 1986.

39. Wei, M. H.; Li, B.; David, R. L. A.; Jones, S. C.; Sarohia, V.; Schmitigal, J. A.; Kornfield, J. A., Science 2015, 350 (6256), 7275.

40. Levene, Stephen D.; Giovan, Stefan M.; Hanke, A.; Shoura, Massa J., Biochem. Soc. Trans. 2013, 41 (2), 513-518.

41. Jacobson, H.; Stockmayer, W. H., J. Chem. Phys. 1950, 18 (12), 1600-1606.

42. Wang, Z. G., Macromolecules 2017, 50 (23), 9073-9114

43. Semenov, A. N.; Nyrkova, I. A.; Cates, M. E., Macromolecules 1995, 28 (23), 7879-7885. 
44. Rubinstein, M.; Colby, R. H., Polymer Physics. Oxford University Press: 2003.

45. Sprague, B. L.; Pego, R. L.; Stavreva, D. A.; McNally, J. G., Biophys. J. 2004, 86 (6), 3473-3495.

46. Soumpasis, D. M., Biophys. J. 1983, 41 (1), 95-97.

47. Rouse, P. E., J. Chem. Phys. 1953, 21 (7), 1272-1280.

48. Zimm, B. H., J. Chem. Phys. 1956, 24 (2), 269-278.

49. De Gennes, P. G., J. Chem. Phys. 1971, 55 (2), 572-579.
50. Miller, W. G.; Goebel, C. V., Biochemistry 1968, 7 (11), 39253935.

51. DeBolt, L. C.; Mark, J. E., Polymer 1987, 28 (3), 416-422.

52. Mandelkern, L.; Mattice, W. L., Biochemistry 1971, 10 (10), 1934-1942.

53. Hassouneh, W.; Zhulina, E. B.; Chilkoti, A.; Rubinstein, M., Macromolecules 2015, 48 (12), 4183-4195. 


\section{GRAPHICAL ABSTRACT / TOC}

local network structure

disallows bridges for $\boldsymbol{R}<R_{\text {mesh }}$

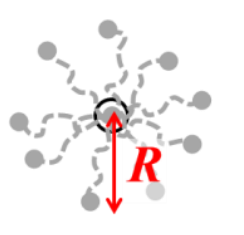

promotes chain

hopping

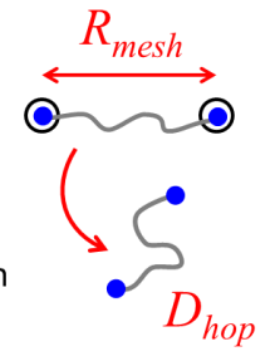

